\title{
COMPLETE MINIMAL SURFACES DERIVED FROM CHEN-GACKSTATTER GENUS TWO EXAMPLE
}

\author{
F.J. López, F. Martín And D. Rodríguez
}

In this paper we present a family of complete minimal surfaces in $\mathbb{R}^{3}$ with one end, arbitrary even genus $k, 4 k$ symmetries and total curvature $-4 \pi(2 k-1)$. Furthermore if $k \neq 6$ they are the only examples satisfying these conditions. If $k=2$ our construction leads to the Chen-Gackstatter genus two surface.

\section{Introduction.}

In 1982 Chen and Gackstatter [C-G] discovered two minimal surfaces with finite total curvature in $\mathbb{R}^{3}$ and non-trivial topology. These examples have only one end and the symmetry of the Enneper's surface.

The first one is the only complete minimal torus in $\mathbb{R}^{3}$ with total curvature $-8 \pi$ (see $[\mathbf{B}],[\mathbf{L} \mathbf{1}]$ ). The second Chen-Gackstatter example has genus two, total curvature $-12 \pi$ and only one end. D. Hoffman noted that these surfaces represented examples with the least absolute total curvature $4(k+1) \pi$ for surfaces with fixed genus $k$, and conjectured that there should be such examples of every genus. Recently Thayer $[\mathbf{T}]$ gave numerical evidence for this conjecture, and Weber and Wolf $[\mathbf{W W}]$ and Sato $[\mathbf{S}]$ proved this conjecture using very different methods. Thus, the main issue here is to obtain uniqueness results for these kind of surfaces.

On the other hand, the classical Chen-Gackstatter examples were generalized in another way, first by Karcher $[\mathbf{K}]$, and later by Thayer [T], Sato [S] (see Martín and Rodríguez work [M-R] for a new approach including uniqueness results), López [L2] and Weber and Wolf $[\mathbf{W W}]$. These authors have shown immersions with only one end and high topology and symmetry.

Except in the case of the Weber and Wolf method, which seems to be very powerful, the other constructions have the same spirit of the Hoffman and Meeks $[\mathbf{H}-\mathbf{M}]$ generalization of the Costa surface $[\mathbf{C}]$. Though this last method is classical and explicit, it has the advantage of providing some natural uniqueness results (see, for instance, $[\mathbf{H}-\mathbf{M}]$ ).

In this paper we use these ideas to show a new family of complete minimal surfaces $M_{k}$ of genus $k, k \geq 2, k$ even, that generalizes the Chen-Gackstatter genus two example. Except in the case of $k=2$ which corresponds to the 
Chen-Gackstatter example, our examples do not lie in any of the families of surfaces discovered by Thayer, Sato and Weber-Wolf. Furthermore, our main achievement is to prove a uniqueness result for these surfaces under some symmetry assumptions.

Summarizing, the surfaces $M_{k}$ have the following properties:

(i) $\quad M_{k}$ is conformally equivalent to $\bar{M}_{k}-\{P\}$, where $\bar{M}_{k}$ is a compact Riemann surface of genus $k$ and $P \in \bar{M}_{k}$.

(ii) $\quad M_{k}$ has total curvature $-4(2 k-1) \pi$.

(iii) $M_{k}$ has $4 k$ symmetries.

(iv) $M_{k}$ intersects the $\left(x_{1}, x_{2}\right)$-plane in $k$ straight lines meeting at equal angles at the origin. Moreover the symmetry group $\operatorname{Sym}\left(M_{k}\right)$ is generated by a rotation by angle $\pi / k$ around the $x_{3}$-axis followed by a symmetry with respect to the $\left(x_{1}, x_{2}\right)$-plane and a symmetry with respect to the $\left(x_{1}, x_{3}\right)$-plane.

(v) $\quad M_{2}$ is the genus two Chen-Gackstatter example.

Moreover, if $M$ is a complete minimal surface with one end, genus $k \neq$ 6 , 15, finite total curvature and $\sharp(\operatorname{Sym}(M))=4 k$, then the total curvature $\mathcal{C}(M)$ of $M$ satisfies $\mathcal{C}(M) \leq-4(2 k-1) \pi$, and the equality holds if and only if $k$ is even and $M$ is, up to rigid motions and scaling, the surface $M_{k}$.

The most important consequence of this is the following uniqueness theorem for the Chen-Gackstatter genus two example:

The Chen-Gackstatter genus two surface is the only complete minimal immersion in $\mathbb{R}^{3}$ of genus two, total curvature $-12 \pi$ and eight symmetries.

The hypothesis $k \neq 6,15$ above may seem to be unnatural. However, as a consequence of elementary topological and algebraical arguments (see the proof of Theorem 2 and Remark 1), it is in fact necessary.

This paper is laid out as follows:

In Section 2 we recall some basic facts about minimal surfaces, emphasizing the classical Weierstrass representation of minimal surfaces and the results of Osserman and Jorge-Meeks.

In Section 3 we state our main existence result Theorem 1, and then we obtain some technical lemmas in order to prove it.

Finally, in Section 4 we obtain the uniqueness result for the surfaces $M_{k}$ mentioned above.

\section{Preliminaries.}

In this section we briefly review some of the results about complete minimal surfaces of finite total curvature.

Let $x: M \longrightarrow \mathbb{R}^{3}$ be a minimal immersion of an orientable surface $M$ 
in three dimensional Euclidean space. Write $\mathcal{C}(M)$ the total curvature of $x$. Using isothermal parameters, $M$ has a conformal structure in a natural way and we label $(g, \eta)$ the Weierstrass data of $x$. Remember that the Gauss map $g$ of $x$ is a meromorphic function on $M$, and $\eta$ is a holomorphic 1-form on $M$ (for more details see $[\mathrm{O}]$ ).

Moreover, $x=\operatorname{Real} \int\left(\phi_{1}, \phi_{2}, \phi_{3}\right)$ where

$$
\phi_{1}=\frac{1}{2} \eta\left(1-g^{2}\right), \phi_{2}=\frac{i}{2} \eta\left(1+g^{2}\right), \phi_{3}=\eta g
$$

are holomorphic 1-forms on $M$ satisfying

$$
\sum_{j=1}^{3}\left|\phi_{j}\right|^{2} \neq 0 .
$$

In particular, $\phi_{j}, j=1,2,3$, have no real periods on $M$.

In the remaining part of this section we suppose $M$ is complete and $\mathcal{C}(M)>-\infty$. Under these assumptions, A. Huber proved (see $[\mathbf{H}]$ ) that $M$ is conformally diffeomorphic to a compact Riemann surface $\bar{M}$ punctured in a finite number of points $\left\{P_{1}, \ldots, P_{r}\right\}$ and R. Osserman $[\mathrm{O}]$ showed that $(g, \eta)$ extends meromorphically to $\bar{M}$. Therefore, $g$ has well defined degree and $\mathcal{C}(M)=-4 \pi \operatorname{deg}(g)$.

Jorge and Meeks $[\mathbf{J}-\mathbf{M}]$ proved that the asymptotic behavior of $x$ around an end $P_{i}$ is determined by the number:

$$
\nu_{i}=\operatorname{Maximum}\left\{\operatorname{ord}\left(\phi_{j}, P_{i}\right), \quad j=1,2,3\right\}-1
$$

where $\operatorname{ord}\left(\phi_{j}, P_{i}\right)$ is the pole order of $\phi_{j}$ at $P_{i}$.

Moreover,

$$
2 \operatorname{deg}(g)=-\chi(\bar{M})+\sum_{i=1}^{r}\left(\nu_{i}+1\right)
$$

Assume that $M$ is not the covering of any minimal surface and write Iso $(M)$ the isometry group of $M$. Then, denote by $\operatorname{Sym}(M)$ the subgroup of Iso $(M)$ which consists of those isometries which are the restriction of a rigid motion in $\mathbb{R}^{3}$ leaving $x(M)$ invariant. Calabi proved that $\operatorname{Iso}(M)=\operatorname{Sym}(M)$ if and only if there exists $j \in\{1,2,3\}$ such that $\phi_{j}$ is not exact. A complete discussion about this subject can be found in $[\mathbf{H}-\mathbf{M}]$.

Through the paper $\mathcal{D}(n)$ denotes the dihedral group of order $2 n$.

We will need the following topological remarks. Let $\bar{M}$ be a compact Riemann surface of genus $k>0$. Given $c_{1}, c_{2} \in H_{1}(M, \mathbb{Z})$, we label $c_{1} \cdot c_{2}$ as the intersection number of $c_{1}$ and $c_{2}$. Consider $\mathcal{B}=\left\{a_{1}, \ldots, a_{k}, b_{1}, \ldots, b_{k}\right\}$ 
a homology basis of $\bar{M}$. Denote the intersection matrix of $\mathcal{B}$ by $D_{\mathcal{B}}=$ $\left(d_{i j}\right)_{1 \leq i, j \leq 2 k}$, where

$$
d_{i j}= \begin{cases}a_{i} \cdot a_{j} & i, j \leq k \\ a_{i} \cdot b_{j-k} & i \leq k<j \\ a_{i-k} \cdot b_{j} & j \leq k<i \\ b_{i-k} \cdot b_{j-k} & i, j>k\end{cases}
$$

By definition, $\mathcal{B}$ is a canonical homology basis if and only if

$$
D_{\mathcal{B}}=\left(\begin{array}{c|c}
0 & I_{k} \\
\hline-I_{k} & 0
\end{array}\right) .
$$

For the details see $[\mathbf{F}-\mathbf{K}]$.

We conclude these preliminaries by recalling the definitions of Euler beta and gamma functions.

For $v \in \mathbb{N}$ and $z \in \mathbb{C}-\{-1,-2, \ldots\}$, the gamma function is given by

$$
\Gamma(z)=\lim _{v \rightarrow+\infty} \frac{v ! v^{z}}{z(z+1)(z+2) \cdots(z+v)} .
$$

Among classical properties of gamma function, we emphasize the following

$$
\begin{gathered}
\Gamma(z+1)=z \Gamma(z), \quad \Gamma(z) \Gamma(1-z)=\frac{\pi}{\sin (\pi z)}, \\
2^{2 z-1} \Gamma(z) \Gamma\left(z+\frac{1}{2}\right)=\sqrt{\pi} \Gamma(2 z) .
\end{gathered}
$$

For $m, n \in \mathbb{C}, \operatorname{Re}(m)>0, \operatorname{Re}(n)>0$, the beta function is defined by

$$
\mathfrak{B}(m, n)=\int_{0}^{1} t^{m-1}(1-t)^{n-1} d t .
$$

This is related to the gamma function according to

$$
\mathfrak{B}(m, n)=\frac{\Gamma(m) \Gamma(n)}{\Gamma(m+n)} .
$$

A complete reference for these topics is, for instance, [Str].

\section{The new family of examples.}

In this section, we present a family of complete orientable minimal surfaces with one end, arbitrary even genus $k \geq 2$ and high symmetry. If $k=2$, then 
this construction leads to the Chen-Gackstatter genus two example. We also give an analytic uniqueness Theorem (Theorem 1) for these surfaces.

Let $\bar{M}_{k a}, k \in \mathbb{N}, k$ even, $k \geq 2, a \in \mathbb{R}_{+}-\{1\}$, be the compact Riemann surface:

$$
\bar{M}_{k a}=\left\{(t, w) \in(\mathbb{C} \cup \infty)^{2}: w^{2}=\frac{t\left(t^{k}-a^{k}\right)}{t^{k}-1}\right\}
$$

and label $\infty=(\infty, \infty), 0=(0,0), e^{\frac{2 j \pi i}{k}}=\left(e^{\frac{2 j \pi i}{k}}, \infty\right), e^{\frac{2 j \pi i}{k}} a=\left(e^{\frac{2 j \pi i}{k}} a, 0\right)$, $j=0, \ldots, k-1$.

We want to define proper minimal immersions of $M_{k a}=\bar{M}_{k a}-\{\infty\}$ into $\mathbb{R}^{3}$, for every $k \geq 2$ ( $k$ even) and for $a \in \mathbb{R}_{+}-\{1\}$, depending on $k$. Consider the following Weierstrass data:

$$
g=A t^{k / 2-1} w, \quad \eta g=B t^{k / 2-1} d t, \quad A \in \mathbb{R}, \quad B \in \mathbb{C},|B|=1
$$

on $\bar{M}_{k a}$. Then, defining $\phi_{j}, j=1,2,3$ as in (1), the inequality (2) is satisfied. Therefore, as we have mentioned in Section 2 , if $\phi_{j}, j=1,2,3$, have no real periods, we get a minimal immersion $x: M_{k a} \rightarrow \mathbb{R}^{3}$ as follows:

$$
x=\text { Real } \int\left(\phi_{1}, \phi_{2}, \phi_{3}\right) \text {. }
$$

The main achievement of this section is the following:

Theorem 1. For each $k \geq 2$, ( $k$ even), there exists only one $a_{0} \in \mathbb{R}_{+}-$ $\{1\}$, depending on $k$, such that $x: M_{k a_{0}} \rightarrow \mathbb{R}^{3}$ is well-defined for a suitable choice of the constants $A, B$.

To prove this theorem, we need to introduce some notations, make some topological comments and obtain several technical analytical lemmas.

The topological part consists of describing a homology basis that allows us to simplify the period problem.

The analytical part consists of studying the behavior of the period functions associated to the above Weierstrass data as functions of the parameter a. This study includes the asymptotic behavior of these functions (Lemma 1 ), the classical bilinear relations (Lemma 2) and some other analytical properties (Lemmas 3, 4, 5, 6).

First, define the following mappings:

$$
\begin{gathered}
J, S: \bar{M}_{k a} \longrightarrow \bar{M}_{k a} \\
J(t, w)=\left(e^{\frac{2 \pi i}{k}} t, e^{\frac{\pi i}{k}} w\right) \quad S(t, w)=(\bar{t}, \bar{w}) .
\end{gathered}
$$


Note that $J$ is holomorphic and has order $2 k$, and $S$ is an antiholomorphic involution. So, they generate a group which is isomorphic to $\mathcal{D}(2 k)$. Moreover, $J$ and $S$ fix $0, \infty$, and $J^{k}$ fixes $e^{\frac{2 j \pi i}{k}}, e^{\frac{2 j \pi i}{k}} a,=j=0, \ldots, k-1$.

Concerning to the topologycal part, we are looking for a homology basis of $\bar{M}_{k a}$. We distinguish two cases:

- Suppose $a>1$. Let $\alpha_{j}(s), \beta_{j}(s), j=1,2$, be the oriented simple closed curves in the $t$-plane illustrated in Figure 1 . We assume that $\alpha_{1}(0) \in \mathbb{R}$, $\alpha_{1}(0)>a, \alpha_{2}(0) \in \mathbb{R}, 1>\alpha_{2}(0)>0, \beta_{1}(0) \in \mathbb{R}, 0>\beta_{1}(0)>-1$, $\beta_{2}(0) \in \mathbb{R}, a>\beta_{2}(0)>1$. Let $a_{j}(s)$ be the unique lift of $\alpha_{j}(s)$ to $\bar{M}_{k a}$ satisfying $w\left(a_{j}(0)\right) \in \mathbb{R}_{+}, j=1,2$. Denote in the same way as $b_{j}(s)$ the corresponding lifts of $\beta_{j}(s)$ with initial conditions $w\left(b_{j}(0)\right) \in i \mathbb{R}_{+}$, $j=1,2$.

- Suppose $0<a<1$. Now, $\alpha_{j}(s), \beta_{j}(s), j=1,2$, are the oriented simple closed curves in the $t$-plane of Figure 2. Here $\alpha_{1}(0) \in \mathbb{R}, \alpha_{1}(0)>1$, $\alpha_{2}(0) \in \mathbb{R}, a>\alpha_{2}(0)>0, \beta_{1}(0) \in \mathbb{R}, 0>\beta_{1}(0)>-a, \beta_{2}(0) \in \mathbb{R}$, $1>\beta_{2}(0)>a$. Let $a_{j}(s)$ be the unique lift of $\alpha_{j}(s)$ to $\bar{M}_{k a}$ satisfying $w\left(a_{j}(0)\right) \in \mathbb{R}_{+}, j=1,2$. Denote in the same way as $b_{1}(s)$ the lift of $\beta_{1}(s)$, with initial condition $w\left(b_{1}(0)\right) \in i \mathbb{R}_{+}$. Define also $b_{2}(s)$ the unique lift of $\beta_{2}(s)$ such that $w\left(b_{2}(s)\right) \in i \mathbb{R}_{-}$.

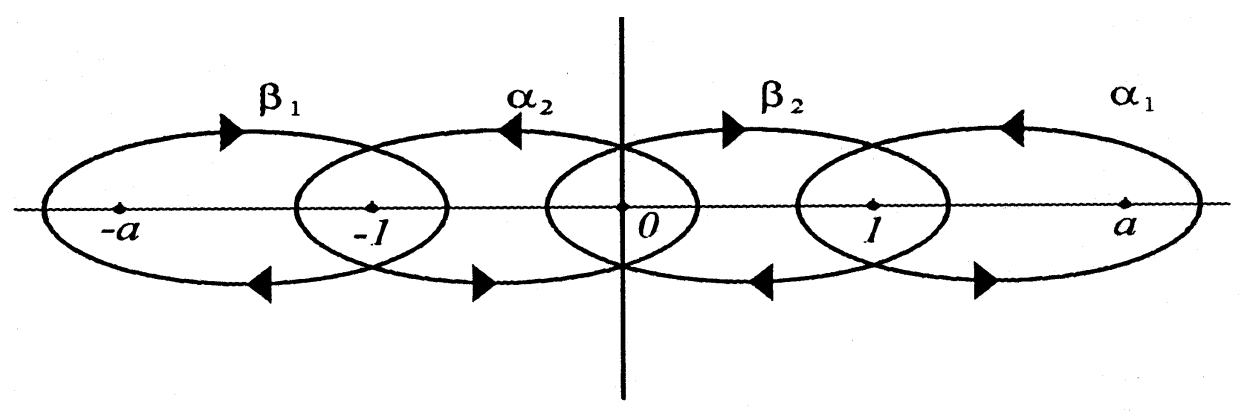

Figure 1. $a>1$. 


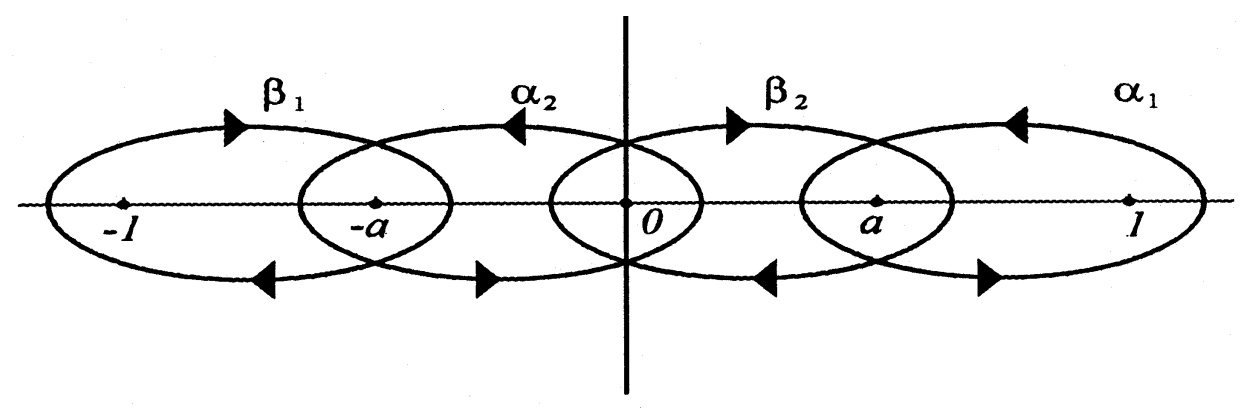

Figure 2. $0<a<1$.

In the following we identify $d$ and its homology class $[d]$, for any closed curve $d$ in $\bar{M}_{k a}$. Then observe that

$$
J_{*}^{k / 2}\left(b_{i}\right)=a_{i}, \quad i=1,2 .
$$

If we label $a_{i}^{j}=\left(J^{j}\right)_{*}\left(a_{i}\right), b_{i}^{j}=\left(J^{j}\right)_{*}\left(b_{i}\right)\left(i=1,2 ; j=0, \ldots, \frac{k}{2}-1\right)$, then

$$
\mathcal{B}=\left\{a_{i}^{j}, b_{i}^{j} \quad i=1,2 \quad j=0, \ldots, \frac{k}{2}-1\right\}
$$

is a homology basis on $\bar{M}_{k a}$ and the intersection matrix $D_{\mathcal{B}}$ is

$a_{1}^{i}$
$a_{2}^{i}$
$b_{2}^{i}$
$b_{1}^{i}$$\left(\begin{array}{r|r|r|r}a_{1}^{j} & a_{2}^{j} & b_{2}^{j} & b_{1}^{j} \\ \hline 0 & 0 & I & 0 \\ \hline-I & G & -G & I \\ \hline 0 & -I & 0 & 0\end{array}\right)$

where $E=\left(e_{i j}\right)_{1 \leq i, j \leq k / 2}, G=\left(g_{i j}\right)_{1 \leq i, j \leq k / 2}$ are given:

$$
e_{i j}=\left\{\begin{array}{rl}
1 & i<j \\
-1 & i>j, \quad g_{i j}=1, \forall i, j \\
0 \quad i & =j
\end{array}\right.
$$

and $I$ is the identity matrix of order $\frac{k}{2}$.

Let $\tau_{1}, \tau_{2}, \sigma_{1}, \sigma_{2}$ be the following 1 -forms on $\bar{M}_{k a}$

$$
\tau_{1}=\frac{d t}{w}, \quad \tau_{2}=t^{k-2} w d t, \quad \sigma_{1}=\frac{d t}{\left(t^{k}-1\right) w}, \quad \sigma_{2}=\frac{t^{k-1} d t}{\left(t^{k}-1\right) w} .
$$

Observe

$$
\begin{aligned}
\phi_{1} & =\frac{B}{2 A}\left(\tau_{1}-A^{2} \tau_{2}\right) \\
\phi_{2} & =\frac{i B}{2 A}\left(\tau_{1}+A^{2} \tau_{2}\right)
\end{aligned}
$$


and $\phi_{3}$ is exact.

So the period problem associated to $\left(\phi_{1}, \phi_{2}, \phi_{3}\right)$ deals with the following functions on $\mathbb{R}_{+}-\{1\}$

$$
f_{1}(a)=\frac{1}{2} \int_{b_{1}} \tau_{1}, \quad f_{2}(a)=\frac{1}{2} \int_{b_{2}} \tau_{1}, \quad g_{1}(a)=\frac{1}{2} \int_{b_{1}} \tau_{2}, \quad g_{2}(a)=\frac{1}{2} \int_{b_{2}} \tau_{2} .
$$

The 1-forms $\sigma_{1}$ and $\sigma_{2}$ and their period functions:

$$
h_{1}(a)=\frac{1}{2} \int_{b_{1}} \sigma_{1}, h_{2}(a)=-\frac{1}{2} \int_{b_{2}} \sigma_{1}, k_{1}(a)=-\frac{1}{2} \int_{b_{1}} \sigma_{2}, k_{2}(a)=-\frac{1}{2} \int_{b_{2}} \sigma_{2}
$$

they will occur naturally by deriving the 1 -forms $\tau_{1}, \tau_{2}$ and the functions $f_{i}, g_{i}, i=1,2$, with respect to parameter $a$.

It is not hard to see that

$$
J^{*}\left(\tau_{1}\right)=e^{\frac{\pi i}{k}} \tau_{1}, J^{*}\left(\tau_{2}\right)=e^{\frac{-\pi i}{k}} \tau_{2}, J^{*}\left(\sigma_{1}\right)=e^{\frac{\pi i}{k}} \sigma_{1}, J^{*}\left(\sigma_{2}\right)=e^{\frac{-\pi i}{k}} \sigma_{2} .
$$

Observe that $a>1$ yields $f_{i}(a), g_{i}(a), h_{i}(a), k_{i}(a)>0 i=1,2$, and $a<1$ implies $f_{1}(a), g_{1}(a)<0, f_{2}(a), g_{2}(a)>0, h_{i}(a), k_{i}(a)>0, i=1,2$. From (4)

$$
\begin{aligned}
\int_{a_{i}} \tau_{1}=2 i f_{i}(a), & \int_{a_{i}} \tau_{2}=-2 i g_{i}(a), \\
\int_{a_{i}} \sigma_{2} & =2 i k_{i}(a), \quad \int_{a_{i}} \sigma_{1}=(-1)^{i+1} 2 i h_{i}(a), \quad i=1,2 .
\end{aligned}
$$

In the remainder of this paper, we denote $f_{3}=\cot \left(\frac{\pi}{2 k}\right) f_{1}+2 f_{2}, g_{3}=$ $\cot \left(\frac{\pi}{2 k}\right) g_{1}+2 g_{2}, h_{3}=\cot \left(\frac{\pi}{2 k}\right) h_{1}-2 h_{2}, k_{3}=\cot \left(\frac{\pi}{2 k}\right) k_{1}+2 k_{2}$.

As we have mentioned above, to prove Theorem 1 we need to do a careful analytical study of the functions $f_{i}, g_{i}, i=1,2$ and their derivates. For this, the following technical lemmas will be useful.

Lemma 1. The asymptotic behavior of $f_{i}, g_{i}, k_{i}, i=1,2$ at $0,1, \infty$ is given as follows:

(i)

$$
\begin{array}{ll}
\lim _{a \rightarrow 0} f_{1}(a) a^{\frac{k-1}{2}}=-\frac{1}{k} \mathfrak{B}\left(\frac{1}{2}, \frac{k-1}{2 k}\right), & \lim _{a \rightarrow 1} \frac{f_{1}(a)}{a-1}=\frac{\pi}{2}, \\
\lim _{a \rightarrow \infty} f_{1}(a) a^{-\frac{1}{2}}=\frac{1}{k} \mathfrak{B}\left(\frac{1}{2}, \frac{k+1}{2 k}\right), & \lim _{a \rightarrow 0} f_{2}(a) a^{\frac{k-1}{2}}=\frac{1}{k} \mathfrak{B}\left(\frac{1}{2}, \frac{1}{2 k}\right), \\
\lim _{a \rightarrow 1} f_{2}(a)=2, & \lim _{a \rightarrow \infty} f_{2}(a) a^{\frac{k}{2}}=\frac{1}{k} \mathfrak{B}\left(\frac{3}{2}, \frac{1}{2 k}\right) .
\end{array}
$$


(ii)

$$
\begin{array}{ll}
\lim _{a \rightarrow 0} g_{1}(a)=-\frac{1}{k} \mathfrak{B}\left(\frac{1}{2}, \frac{3 k-1}{2 k}\right), & \lim _{a \rightarrow 1} \frac{g_{1}(a)}{a-1}=\frac{\pi}{2}, \\
\lim _{a \rightarrow \infty} g_{1}(a) a^{\frac{1-2 k}{2}}=\frac{1}{k} \mathfrak{B}\left(\frac{3}{2}, \frac{k-1}{2 k}\right), & \lim _{a \rightarrow 0} g_{2}(a) a^{\frac{1-3 k}{2}}=\frac{1}{k} \mathfrak{B}\left(\frac{3}{2}, \frac{2 k-1}{2 k}\right), \\
\lim _{a \rightarrow 1} g_{2}(a)=\frac{2}{2 k-1}, & \lim _{a \rightarrow \infty} g_{2}(a) a^{-\frac{k}{2}}=\frac{1}{k} \mathfrak{B}\left(\frac{1}{2}, \frac{2 k-1}{2 k}\right) .
\end{array}
$$

$$
\begin{array}{ll}
\lim _{a \rightarrow 0} k_{1}(a)=\frac{1}{k} \mathfrak{B}\left(\frac{1}{2}, \frac{k-1}{2 k}\right), & \lim _{a \rightarrow \infty} k_{1}(a) a^{\frac{1}{2}}=\frac{1}{k} \mathfrak{B}\left(\frac{1}{2}, \frac{k-1}{2 k}\right), \\
\lim _{a \rightarrow 0} k_{2}(a) a^{\frac{1-k}{2}}=\frac{1}{k} \mathfrak{B}\left(\frac{1}{2}, \frac{2 k-1}{2 k}\right), & \lim _{a \rightarrow \infty} k_{2}(a) a^{\frac{k}{2}}=\frac{1}{k} \mathfrak{B}\left(\frac{1}{2}, \frac{2 k-1}{2 k}\right),
\end{array}
$$

where $\mathfrak{B}$ is the classical Beta Function.

Proof. From the definition of $f_{1}$ it follows that

$$
f_{1}(a)=\int_{1}^{a} \sqrt{\frac{t^{k}-1}{t\left(a^{k}-t^{k}\right)}} d t .
$$

Several changes of variables give

$$
\begin{aligned}
f_{1}(a) & =\int_{1}^{a} \sqrt{\frac{t^{k}-1}{t\left(a^{k}-t^{k}\right)}} d t=\frac{1}{k} \int_{1}^{a^{k}} u^{\frac{1-2 k}{2 k}} \sqrt{\frac{u-1}{a^{k}-u}} d u \\
& =\frac{1}{k}\left(a^{k}-1\right) \int_{0}^{1} \frac{\left(\left(a^{k}-1\right) s+1\right)^{\frac{1-2 k}{2 k}} \sqrt{s}}{\sqrt{1-s}} d s .
\end{aligned}
$$

Hence,

$$
\lim _{a \rightarrow 1} \frac{f_{1}(a)}{a-1}=\int_{0}^{1} \sqrt{\frac{s}{1-s}} d s=\frac{\pi}{2} .
$$

Using that $\lim _{a \rightarrow \infty} f_{1}(a)=\lim _{b \rightarrow 0} f_{1}\left(\frac{1}{b}\right)$ we obtain

$$
\begin{aligned}
\lim _{a \rightarrow \infty} f_{1}(a) a^{\frac{-1}{2}} & =\lim _{b \rightarrow 0} \frac{1-b^{k}}{k} \int_{0}^{1} \frac{\left(\left(1-b^{k}\right) s+b^{k}\right)^{\frac{1-2 k}{2 k}} \sqrt{s}}{\sqrt{1-s}} d s \\
& =\frac{1}{k} \int_{0}^{1} s^{\frac{1-k}{2 k}}(1-s)^{-\frac{1}{2}} d s=\frac{1}{k} \mathfrak{B}\left(\frac{1}{2}, \frac{k+1}{2 k}\right) .
\end{aligned}
$$


For computing the limit at 0 we need another change of variable. First, put $t=\frac{1}{x}$ in the expression of $f_{1}$ and obtain

$$
\begin{aligned}
f_{1}(a) & =-\int_{1}^{\frac{1}{a}} \frac{\sqrt{x^{k}-1}}{x^{\frac{3}{2}} \sqrt{1-a^{k} x^{k}}} d x=-\frac{1}{k} \int_{1}^{\frac{1}{a^{k}}} \frac{\sqrt{u-1}}{u^{\frac{1+2 k}{2 k}} \sqrt{1-a^{k} u}} d u \\
& =\frac{a^{\frac{1-k}{2}}\left(a^{k}-1\right)}{k} \int_{0}^{1} \frac{\sqrt{s}}{\sqrt{1-s}\left(a^{k}+s\left(1-a^{k}\right)\right)^{\frac{1+2 k}{2 k}}} d s
\end{aligned}
$$

then

$$
\lim _{a \rightarrow 0} a^{\frac{k-1}{2}} f_{1}(a)=-\frac{1}{k} \int_{0}^{1} s^{-\frac{k+1}{2 k}}(1-s)^{-\frac{1}{2}} d s=\frac{1}{k} \mathfrak{B}\left(\frac{1}{2}, \frac{k-1}{2 k}\right) .
$$

Similar arguments and changes of variables complete the above assertions for $g_{i}$ and $k_{i}, i=1,2$.

Lemma 2. The functions $f_{i}, g_{i}, h_{i}, k_{i}, i \in\{1,3\}$ satisfy:

(i) $f_{1} g_{3}+f_{3} g_{1}=\frac{4 \pi\left(a^{k}-1\right)}{2 k-1}$,

(ii) $f_{1} k_{3}+f_{3} k_{1}=\frac{4 \pi}{k}$,

(iii) $g_{1} h_{3}+g_{3} h_{1}=\frac{4 \pi}{k(2 k-1)}$,

(iv) $h_{1} k_{3}+h_{3} k_{1}=0$.

Proof. Using classical bilinear relations we obtain

$$
-\vec{v}_{1} \cdot D_{\mathcal{B}}^{-1} \cdot{ }^{t} \vec{v}_{2}=2 \pi i \text { Residue }\left(f \tau_{2}, \infty\right)
$$

where

$$
\begin{gathered}
\vec{v}_{1}=\left(\int_{a_{1}^{0}} \tau_{1}, \ldots, \int_{a_{1}^{k / 2-1}} \tau_{1}, \int_{a_{2}^{0}} \tau_{1}, \ldots, \int_{a_{2}^{k / 2-1}} \tau_{1},\right. \\
\left.\int_{b_{2}^{0}} \tau_{1}, \ldots, \int_{b_{2}^{k / 2-1}} \tau_{1}, \int_{b_{1}^{0}} \tau_{1}, \ldots, \int_{b_{1}^{k / 2-1}} \tau_{1}\right) \\
\vec{v}_{2}=\left(\int_{a_{1}^{0}} \tau_{2}, \ldots, \int_{a_{1}^{k / 2-1}} \tau_{2}, \int_{a_{2}^{0}} \tau_{2}, \ldots, \int_{a_{2}^{k / 2-1}} \tau_{2},\right. \\
\left.\int_{b_{2}^{0}} \tau_{2}, \ldots, \int_{b_{2}^{k / 2-1}} \tau_{2}, \int_{b_{1}^{0}} \tau_{2}, \ldots, \int_{b_{1}^{k / 2-1}} \tau_{2}\right) \\
D_{\mathcal{B}}^{-1}=\left(\begin{array}{r|r|r|r}
E & 0 & -I & -G \\
\hline 0 & 0 & 0 & -I \\
\hline I & 0 & 0 & 0 \\
\hline G & I & 0 & E
\end{array}\right)
\end{gathered}
$$


and $\tau_{1}=d f$ locally around $\infty$. Taking into account (4) and (5) we conclude that

$$
k i\left[\cot \left(\frac{\pi}{2 k}\right) \int_{b_{1}} \tau_{1} \int_{b_{1}} \tau_{2}+\int_{b_{1}} \tau_{1} \int_{b_{2}} \tau_{2}+\int_{b_{2}} \tau_{1} \int_{b_{1}} \tau_{2}\right]=2 \pi i \frac{4 k\left(a^{k}-1\right)}{2 k-1}
$$

and using the definitions of the functions $f_{i}, g_{i}, i \in\{1,3\}$ it is not hard to check (i). Applying the same argument to the pairs $\left(\tau_{1}, \sigma_{2}\right),\left(\tau_{2}, \sigma_{1}\right)$ and $\left(\sigma_{1}, \sigma_{2}\right)$ we obtain the equalities (ii), (iii) and (iv), respectively.

Lemma 3. The following equalities hold:

(i) $\frac{d f_{i}}{d a}=\frac{1}{2 a} f_{i}+\frac{k}{2 a} h_{i}$,

(ii) $\frac{d g_{i}}{d a}=\frac{k a^{k-1}}{2} k_{i}$,

(iii) $\frac{d h_{i}}{d a}=\frac{1}{2 a\left(a^{k}-1\right)} f_{i}-\frac{k}{2 a} h_{i}$,

(iv) $\frac{d k_{i}}{d a}=\frac{2 k-1}{2 a\left(1-a^{k}\right)} g_{i}+\frac{k-1}{2 a} k_{i}$,

for $i \in\{1,3\}$.

Proof. Formally,

$$
\begin{aligned}
\frac{d \tau_{1}}{d a} & =\frac{1}{2 a} \tau_{1}+\frac{k}{2 a} \sigma_{1}-\frac{1}{a} d\left(\frac{t}{w}\right), \quad \frac{d \tau_{2}}{d a}=-\frac{k a^{k-1}}{2} \sigma_{2}, \\
\frac{d \sigma_{1}}{d a} & =\frac{1}{2 a\left(a^{k}-1\right)} \tau_{1}-\frac{k}{2 a} \sigma_{1}+\frac{1}{a\left(1-a^{k}\right)} d\left(\frac{t}{w}\right), \\
\frac{d \sigma_{2}}{d a} & =\frac{2 k-1}{2 a\left(a^{k}-1\right)} \tau_{2}+\frac{k-1}{2 a} \sigma_{2}+\frac{1}{a\left(1-a^{k}\right)} d\left(\frac{t^{k}}{w}\right) .
\end{aligned}
$$

Integrating on the suitable curves, it is easy to deduce the statements of this lemma.

Lemma 4. The following equations hold:

(i) $h_{1} f_{3}-h_{3} f_{1}=\frac{4 \pi}{k} a^{\frac{1-k}{2}}$,

(ii) $g_{1} k_{3}-g_{3} k_{1}=-\frac{4 \pi}{k(2 k-1)} a^{\frac{k-1}{2}}$.

Proof. From Lemma 3, we can check the following equations

$$
\begin{aligned}
\frac{d}{d a}\left(h_{1} f_{3}-h_{3} f_{1}\right) & =\frac{1-k}{2 a}\left(h_{1} f_{3}-h_{3} f_{1}\right), \\
\frac{d}{d a}\left(g_{1} k_{3}-g_{3} k_{1}\right) & =\frac{k-1}{2 a}\left(g_{1} k_{3}-g_{3} k_{1}\right) .
\end{aligned}
$$


If we integrate these ordinary differential equations then we obtain

$$
\begin{aligned}
& h_{1}(a) f_{3}(a)-h_{3}(a) f_{1}(a)= \begin{cases}C_{1} a^{\frac{1-k}{2}} & \text { if } a>1 \\
D_{1} a^{\frac{1-k}{2}} & \text { if } 0<a<1\end{cases} \\
& g_{1}(a) k_{3}(a)-g_{3}(a) k_{1}(a)= \begin{cases}C_{2} a^{\frac{k-1}{2}} & \text { if } a>1 \\
D_{2} a^{\frac{k-1}{2}} & \text { if } 0<a<1 .\end{cases}
\end{aligned}
$$

Hence,

$$
\left(h_{1} f_{3}-h_{3} f_{1}\right)\left(g_{1} k_{3}-g_{3} k_{1}\right)= \begin{cases}C_{1} \cdot C_{2} & \text { if } a>1 \\ D_{1} \cdot D_{2} & \text { if } 0<a<1 .\end{cases}
$$

Expanding and using (ii), (iii) and (iv) in Lemma 2 we conclude $C_{1} \cdot C_{2}=$ $D_{1} \cdot D_{2}=-\frac{16 \pi^{2}}{k^{2}(2 k-1)}$. But using Lemma 1 and the properties of Beta and Gamma Functions, we get

$$
\begin{aligned}
C_{2} & =\lim _{a \rightarrow \infty} \frac{g_{1}(a) k_{3}(a)-g_{3}(a) k_{1}(a)}{a^{\frac{k-1}{2}}}=\lim _{a \rightarrow \infty} 2 \frac{g_{1}(a) k_{2}(a)-g_{2}(a) k_{1}(a)}{a^{\frac{k-1}{2}}} \\
& =\frac{2}{k^{2}}\left[\mathfrak{B}\left(\frac{3}{2}, \frac{k-1}{2 k}\right) \mathfrak{B}\left(\frac{1}{2}, \frac{2 k-1}{2 k}\right)-\mathfrak{B}\left(\frac{1}{2}, \frac{2 k-1}{2 k}\right) \mathfrak{B}\left(\frac{1}{2}, \frac{k-1}{2 k}\right)\right] \\
& =-\frac{4 \pi}{k(2 k-1)}, \\
D_{2} & =\lim _{a \rightarrow 0} \frac{g_{1}(a) k_{3}(a)-g_{3}(a) k_{1}(a)}{a^{\frac{k-1}{2}}}=\lim _{a \rightarrow 0} 2 \frac{g_{1}(a) k_{2}(a)-g_{2}(a) k_{1}(a)}{a^{\frac{k-1}{2}}} \\
& =-\frac{2}{k^{2}} \mathfrak{B}\left(\frac{1}{2}, \frac{3 k-1}{2 k}\right) \mathfrak{B}\left(\frac{1}{2}, \frac{2 k-1}{2 k}\right) \\
& =-\frac{4 \pi}{k(2 k-1)}
\end{aligned}
$$

and so, $C_{1}=D_{1}=\frac{4 \pi}{k}$.

Lemma 5. The functions $f_{1}, f_{3}, g_{1}, g_{3}$ satisfy

$$
\begin{aligned}
& \text { (i) } \frac{d f_{i}}{d a}=\frac{a^{k-1}}{2\left(a^{k}-1\right)} f_{i}(a)+(-1)^{\frac{i-1}{2}} \frac{(2 k-1) a^{-\frac{k+1}{2}}}{2\left(a^{k}-1\right)} g_{i}(a), \\
& \text { (ii) } \frac{d g_{i}}{d a}=(-1)^{\frac{i-1}{2}} \frac{a^{\frac{3(k-1)}{2}}}{2\left(a^{k}-1\right)} f_{i}(a)+\frac{(2 k-1) a^{k-1}}{2\left(a^{k}-1\right)} g_{i}(a),
\end{aligned}
$$
for $i \in\{1,3\}$.

Proof. From Lemmas 2 and 4, observe that the functions $h_{i}, k_{i}$ satisfy the 
following linear systems

$$
\left.\left.\begin{array}{l}
f_{1} k_{3}+f_{3} k_{1}=\frac{4 \pi}{k} \\
g_{1} k_{3}-g_{3} k_{1}=-\frac{4 \pi}{k(2 k-1)} a^{\frac{k-1}{2}}
\end{array}\right\} \begin{array}{l}
g_{1} h_{3}+g_{3} h_{1}=\frac{4 \pi}{k(2 k-1)} \\
-f_{1} h_{3}+f_{3} h_{1}=\frac{4 \pi}{k} a^{\frac{1-k}{2}}
\end{array}\right\} .
$$

Solving and using (i) in Lemma 2, we obtain new expressions for $k_{1}, k_{3}, h_{1}$, $h_{3}$ depending on $f_{1}, f_{3}, g_{1}, g_{3}$. Substituting them in the equalities (i) and (ii) in Lemma 3 we conclude the proof.

Let us define $\varphi: \mathbb{R}_{+}-\{1\} \longrightarrow \mathbb{R}$,

$$
\varphi(a)=\frac{f_{3}(a)}{f_{1}(a)}-\frac{g_{3}(a)}{g_{1}(a)} .
$$

As a consequence of the preceeding analysis we can state the following lemma, which is fundamental in the proof of Theorem 1:

Lemma 6. The funtion $\varphi$ vanishes at a unique point $a_{0} \in \mathbb{R}_{+}-\{1\}$.

Proof. Firstly we study the asymptotic behavior of $\varphi$ at 0,1 and $\infty$. From Lemma 1 we deduce that:

$$
\begin{aligned}
\lim _{a \rightarrow 0} \varphi(a) & =2 \lim _{a \rightarrow 0} \frac{f_{2}(a) g_{1}(a)-f_{1}(a) g_{2}(a)}{f_{1}(a) g_{1}(a)}=-2 \frac{\mathfrak{B}\left(\frac{1}{2}, \frac{1}{2 k}\right)}{\mathfrak{B}\left(\frac{1}{2}, \frac{k-1}{2 k}\right)}<0 \\
\lim _{a \rightarrow 1^{-}} \varphi(a) & =\frac{16(k-1)}{(2 k-1) \pi} \lim _{a \rightarrow 1^{-}} \frac{1}{a-1}=-\infty \\
\lim _{a \rightarrow 1^{+}} \varphi(a) & =\frac{16(k-1)}{(2 k-1) \pi} \lim _{a \rightarrow 1^{+}} \frac{1}{a-1}=+\infty \\
\lim _{a \rightarrow+\infty} \varphi(a) a^{\frac{k-1}{2}} & =\lim _{a \rightarrow+\infty}\left(\frac{1}{a} \frac{f_{2}(a) a^{\frac{k}{2}}}{f_{1}(a) a^{-\frac{1}{2}}}-\frac{g_{2}(a) a^{-\frac{k}{2}}}{g_{1}(a) a^{\frac{1-2 k}{2}}}\right)=-\frac{\mathfrak{B}\left(\frac{1}{2}, \frac{2 k-1}{2 k}\right)}{\mathfrak{B}\left(\frac{3}{2}, \frac{k-1}{2 k}\right)}<0
\end{aligned}
$$

so, there exists $\left.a_{0} \in\right] 1,+\infty\left[\right.$ such that $\varphi\left(a_{0}\right)=0$.

In fact, $\varphi$ has only one zero on $\mathbb{R}_{+}-\{1\}$. To see this we need to compute $\varphi^{\prime}(a)$ and so do a careful analysis of the behavior of $\varphi$. From Lemma 5, we obtain:

$$
\varphi^{\prime}(a)=a^{-\frac{k+1}{2}}\left(a^{2 k-1} f_{1}^{2}(a)-(2 k-1) g_{1}^{2}(a)\right) \frac{f_{3}(a) g_{1}(a)+f_{1}(a) g_{3}(a)}{2\left(a^{k}-1\right) f_{1}^{2}(a) g_{1}^{2}(a)}
$$

and using (i) in Lemma 2 we deduce:

$$
\begin{aligned}
& \varphi^{\prime}(a)= \frac{2 \pi a^{-\frac{k+1}{2}}}{(2 k-1) f_{1}^{2}(a) g_{1}^{2}(a)}\left(a^{\frac{2 k-1}{2}} f_{1}(a)+\sqrt{2 k-1} g_{1}(a)\right) \\
& \cdot\left(a^{\frac{2 k-1}{2}} f_{1}(a)-\sqrt{2 k-1} g_{1}(a)\right) .
\end{aligned}
$$


Labeling

$$
\begin{aligned}
\rho: \mathbb{R}_{+}-\{1\} & \longrightarrow \mathbb{R} \\
\rho(a) & =a^{\frac{2 k-1}{2}} f_{1}(a)-\sqrt{2 k-1} g_{1}(a)
\end{aligned}
$$

using the properties of $f_{1}$ and $g_{1}$ described in page 318 , we observe that $\frac{\varphi^{\prime}}{\rho}$ is positive in $] 1,+\infty[$ and negative in ]0,1[. It follows from Lemma 1 that:

$$
\begin{gathered}
\lim _{a \rightarrow 0} \rho(a)=\frac{\sqrt{2 k-1}}{k} \mathfrak{B}\left(\frac{1}{2}, \frac{3 k-1}{2 k}\right)>0, \\
\lim _{a \rightarrow 1} \rho(a)=0, \lim _{a \rightarrow 1} \rho^{\prime}(a)=\frac{\pi(1-\sqrt{2 k-1})}{2}<0, \\
\lim _{a \rightarrow \infty} \rho(a)=+\infty .
\end{gathered}
$$

Furthermore, if $a_{1} \in \mathbb{R}_{+}-\{1\}$ satisfies $\rho\left(a_{1}\right)=0$ then from Lemma 3

$$
\rho^{\prime}\left(a_{1}\right)=\frac{\sqrt{2 k-1}\left(a_{1}^{k}-(2 k-1)\right) g_{1}\left(a_{1}\right)}{2 a_{1}\left(a_{1}^{k}-1\right)} .
$$

Hence, to obtain a contradiction, suppose that $\left.a_{1} \in\right] 0,1[$. This implies $\rho^{\prime}\left(a_{1}\right)<0$, and since $\rho(1)=0, \rho^{\prime}(1)<0$ we deduce that there exists another point $\left.a_{2} \in\right] 0,1\left[\right.$ such that $a_{2}>a_{1}, \rho\left(a_{2}\right)=0$ and $\rho^{\prime}\left(a_{2}\right)>0$, which is clearly absurd. Thus $\rho(a)>0, \forall a \in] 0,1[$.

Suppose $a_{1}$ is the lowest root of $\rho$ in $] 1,+\infty\left[\right.$. If $\left.a_{1} \in\right] 1, \sqrt[k]{2 k-1}[$ then $\rho^{\prime}\left(a_{1}\right)<0$ which is contrary to the choice of $a_{1}$ and the facts $\rho(1)=$ $0, \rho^{\prime}(1)<0$. Therefore $\left.\rho(a)<0, \forall a \in\right] 1, \sqrt[k]{2 k-1}[$. Assume that $\rho$ has at least three zeroes $a_{1}, a_{2}, a_{3}$ in $[\sqrt[k]{2 k-1},+\infty[$. Without loss of generality, we can suppose $a_{1}<a_{2}<a_{3}$ and these three points are the lowest roots of $\rho$. Then $\left.a_{2}, a_{3} \in\right] \sqrt[k]{2 k-1},+\infty\left[\right.$ and so $\rho^{\prime}\left(a_{2}\right), \rho^{\prime}\left(a_{3}\right)>0$ which is absurd. Thus $\rho$ has at most two zeroes in $] 1,+\infty[$.

The above remarks imply $\left.\varphi^{\prime}(a)<0 \forall a \in\right] 0,1[$ and taking into account the asymptotic behavior of $\varphi$ at 0 and 1 we get $\varphi(a)<0 \forall a \in] 0,1$ [. Analogously, $\varphi^{\prime}$ has at most two zeroes in $] 1,+\infty[$. Assume that $\varphi$ has at least two zeroes in ]1, $+\infty$ [. According to the limits of this function at 1 and $+\infty$ we conclude that $\varphi^{\prime}$ has at least three roots, which is absurd. This contradiction completes the proof.

Proof of Theorem 1. The immersion $x$ is well defined if and only if $\operatorname{Real}\left(\int_{d} \phi_{j}\right)=0$, for every closed curve $d$ in $M_{k a}$ and $j \in\{1,2,3\}$. As $\phi_{j}$ has only one singularity at $\infty$, then $\operatorname{Residue}\left(\phi_{j}, \infty\right)=0, j=1,2,3$. So, it suffices to prove:

$$
\operatorname{Real}\left(\int_{d} \phi_{j}\right)=0, \quad j=1,2,3
$$


for any closed curve, $d$, lying in the homology basis $\mathcal{B}$ of $\bar{M}_{k a}$ defined at the beginning of this section.

If we put $\Phi=\left(\phi_{1}, \phi_{2}, \phi_{3}\right)^{t}$ then $J^{*}(\Phi)=R \cdot \Phi$, where $R \in \mathcal{O}(3)$ is the matrix

$$
R=\left(\begin{array}{ccc}
\cos (\pi / k) & \sin (\pi / k) & 0 \\
-\sin (\pi / k) & \cos (\pi / k) & 0 \\
0 & 0 & -1
\end{array}\right) .
$$

Hence using the last equality and $(4), \operatorname{Real}\left(\int_{d} \Phi\right)=\overrightarrow{0}, \forall d \in \mathcal{B}$ if and only if:

$$
\operatorname{Real}\left(\int_{b_{1}} \Phi\right)=\operatorname{Real}\left(\int_{b_{2}} \Phi\right)=\overrightarrow{0}
$$

Recall that $\phi_{3}$ is exact, $\phi_{1}=\frac{B}{2 A}\left(\tau_{1}-A^{2} \tau_{2}\right)$ and $\phi_{2}=\frac{i B}{2 A}\left(\tau_{1}+A^{2} \tau_{2}\right)$. Thus, using the definitions of $f_{i}, i=1,2$, the last equations hold if and only if $B^{2}=1$ and

$$
\begin{aligned}
& f_{1}(a)=A^{2} g_{1}(a) \\
& f_{2}(a)=A^{2} g_{2}(a)
\end{aligned}
$$

for some $A \in \mathbb{R}, a \in \mathbb{R}_{+}-\{1\}$. As $f_{i}(a), g_{i}(a) \in \mathbb{R}^{*}, \forall a \in \mathbb{R}_{+}-\{1\}$, then the existence of $a, A$ satisfying the former is equivalent to solving the following equation

$$
f_{1}(a) g_{3}(a)-f_{3}(a) g_{1}(a)=0
$$

and putting $A^{2}=\frac{f_{1}(a)}{g_{1}(a)}>0$ (see page 318 ). Recalling the definition of the function $\varphi$, this means that $\varphi(a)=0$. Using Lemma 6 we conclude the proof.

\section{Uniqueness results.}

In this section we obtain some uniqueness theorems for the surfaces arising from Theorem 1. Throughout this section and for the sake of simplicity we denote these surfaces as $M_{k}$ instead of $M_{k a_{0}}$.

Let $x: M \longrightarrow \mathbb{R}^{3}$ be a complete orientable minimal surface with finite total curvature and one end and label $(g, \eta)$ as its Weierstrass data. From Huber and Osserman theorems, there exist a compact Riemann surface $\bar{M}$ and one point $P \in \bar{M}$ such that $M$ is conformally equivalent to $\bar{M}-\{P\}$. We write $k=\operatorname{genus}(\bar{M})$ and assume that $k \geq 2$.

As an easy consequence of Hurwitz's Theorem (see $[\mathbf{F}-\mathbf{K}]$ ), the group $\operatorname{Sym}(M)$ is finite, and so up to a suitable choice of the origin, it is given by a linear group of isometries of $\mathbb{R}^{3}$. 
When $R>0, R$ being big enough, we get $\bar{D}=x^{-1}\left(\left\{\left(x_{1}, x_{2}, x_{3}\right) \in \mathbb{R}^{3}\right.\right.$ : $\left.\left.x_{1}^{2}+x_{2}^{2}+x_{3}^{2} \geq R\right\}\right) \cup\{P\}$ is a conformal disc in $\bar{M}$. Without loss of generality $\bar{D} \equiv \bar{D}(0,1)$ and $P \equiv 0$. Since $\operatorname{Sym}(M)$ leaves $\bar{D}$ invariant and fixes 0 , the group $\Delta=\left\{S_{\mid \bar{D}}: S \in \operatorname{Sym}(M)\right\}$ is either cyclic or generated by a rotation around 0 and a symmetry with respect to a straight line in $\mathbb{C}$ containing 0 (that is, $\Delta$ is isomorphic to the dihedral group $D\left(\frac{d}{2}\right)$, where $d=\sharp(\operatorname{Sym}(M))$ is the cardinal of $\Delta$ ).

Up to rotations, we can suppose $g(P)=\infty$. We denote $J \in \operatorname{Sym}(M)$ as a symmetry whose restriction $J_{\mid \bar{D}}$ generates the subgroup of holomorphic transformations of $\Delta$. It is clear that $\operatorname{ord}(J)$ is either $d$ or $d / 2$. Observe that $J$ extends conformally to $\bar{M}$ and looked at as linear isometry it fixes the $x_{3}$-axis. If $\operatorname{ord}(J)>2$, this linear transformation is either a rotation around the $x_{3}$-axis or a rotation around the $x_{3}$-axis followed by a symmetry with respect to the $\left(x_{1}, x_{2}\right)$-plane. Without loss of generality, we suppose the rotation determined by $J$ is by angle $\frac{2 \pi}{\operatorname{ord}(J)}$. As the normal limit vector of $x$ at $P$ is vertical, the $x_{3}$-axis intersects $x(M)$ in a finite set of points and therefore $J$ fixes a finite set of points of $\bar{M}$. Define, for each $Q \in \bar{M}$, the isotropy group $H_{Q}=\{T \in\langle J\rangle: T(Q)=Q\}$, and the orbit of $Q$ : $\operatorname{orb}(Q)=\left\{Q, J(Q), \ldots, J^{\operatorname{ord}(J)-1}(Q)\right\}$. Note that $\operatorname{orb}(P)=\{P\}$.

Theorem 2. Suppose $\sharp(\operatorname{Sym}(M))=4 k$ and $k \neq 6,15$. Then $\mathcal{C}(M) \leq$ $-4 \pi(2 k-1)$, and the equality holds if and only if $k$ is even and $x: M \rightarrow \mathbb{R}^{3}$ is, up to rigid motions and scaling, the minimal surface $M_{k}$ given in Theorem 1.

Proof. Riemann-Hurwitz formula gives:

$$
2-2 k=\operatorname{ord}(J) \chi\left(\frac{\bar{M}}{\langle J\rangle}\right)-\operatorname{ord}(J)+1-\sum_{Q \in M}(\mu(Q)-1),
$$

where $\mu(Q)=\sharp\left(H_{Q}\right)$.

Since $\operatorname{ord}(J) \in\{2 k, 4 k\}$, then $\chi\left(\frac{\bar{M}}{\langle J\rangle}\right)>0$, and so $\chi\left(\frac{\bar{M}}{\langle J\rangle}\right)=2$ and $\frac{\bar{M}}{\langle J\rangle}=$ $\overline{\mathbb{C}}$. Thus,

$$
\sum_{Q \in M}(\mu(Q)-1)=\operatorname{ord}(J)+2 k-1 .
$$

In particular, there exist some points $Q \in M$ such that $\mu(Q)>1$. Label $u: \bar{M} \rightarrow \overline{\mathbb{C}}=\bar{M} /\langle J\rangle$ as the natural projection. The singular values of $u$ are denoted by $\left\{\alpha_{1}, \ldots, \alpha_{s}\right\} \subset \overline{\mathbb{C}}, s>1$, i.e., $\mu(Q)>1$ for each $Q \in$ $u^{-1}\left(\left\{\alpha_{1}, \ldots, \alpha_{s}\right\}\right)$ and $\mu(Q)=1$ for each $Q \notin u^{-1}\left(\left\{\alpha_{1}, \ldots, \alpha_{s}\right\}\right)$. Choose $Q_{i} \in u^{-1}\left(\alpha_{i}\right)$ and define $m_{i}=\operatorname{ord}(J) / \mu\left(Q_{i}\right), i=1, \ldots, s$. It is clear that 
$m_{i} \in \mathbb{Z}$ and $1 \leq m_{i} \leq \operatorname{ord}(J) / 2, i=1, \ldots, s$. Then,

$$
\begin{aligned}
H_{Q_{i}} & =\left\{J^{n m_{i}}: n=0,1, \ldots, \mu\left(Q_{i}\right)-1\right\} \\
\operatorname{orb}\left(Q_{i}\right) & =u^{-1}\left(\alpha_{i}\right)=\left\{Q_{i}, J\left(Q_{i}\right), \ldots, J^{m_{i}-1}\left(Q_{i}\right)\right\}
\end{aligned}
$$

and so,

$$
\sum_{Q \in M}(\mu(Q)-1)=\sum_{i=1}^{s}\left(\operatorname{ord}(J)-m_{i}\right)
$$

Hence, we deduce

$$
\sum_{i=1}^{s}\left(\operatorname{ord}(J)-m_{i}\right)=\operatorname{ord}(J)+2 k-1 .
$$

From (7) it is obvious that $s \geq 2$. Using $m_{i} \leq \operatorname{ord}(J) / 2, i=1, \ldots, s$, and $\operatorname{ord}(J) \geq 2 k$ we get $s<4$, i.e., $s \in\{2,3\}$.

Given $Q \in \bar{M}$ whose normal vector $g(Q)$ is vertical, it is clear that:

$$
\operatorname{orb}(Q) \subset g^{-1}(g(Q)) .
$$

In order to see this, observe that $g \circ J=\theta g, \theta^{\operatorname{ord}(J)}=1$. Furthermore, if $Q$ is a fixed point of $J^{m_{i}}$ the multiplicity of $Q$ as zero or pole of $g$ is:

- If $J^{m_{i}}$ is a rotation around the $x_{3}$-axis,

$$
\frac{\operatorname{ord}(J)}{m_{i}} l_{i}-1, \text { where } l_{i} \in \mathbb{N} .
$$

- If $J^{m_{i}}$ is a rotation around the $x_{3}$-axis followed by a symmetry with respect to the $\left(x_{1}, x_{2}\right)$-plane,

$$
\frac{\operatorname{ord}(J)}{2 m_{i}} \widetilde{l}_{i}-1, \text { where } \widetilde{l}_{i} \in \mathbb{N}, \widetilde{l}_{i} \text { odd. }
$$

We distinguish two cases: $\operatorname{ord}(J)=2 k, \operatorname{ord}(J)=4 k$.

First case: $\operatorname{ord}(J)=2 k$.

From (7) once again, $s=3$.

At this point, we can describe the underlying complex structure of $\bar{M}$. Up to a Möbius transformation, we put $u(P)=\infty, u\left(Q_{1}\right)=0$, and label $u\left(Q_{2}\right)=c, u\left(Q_{3}\right)=b ; c, b \in \mathbb{C}-\{0\}, b \neq c$.

Since $d=2 \operatorname{ord}(J)=4 k$, there exists an antiholomorphic transformation $S \in \operatorname{Sym}(M)$ satisfying $S^{2}=\operatorname{Id}, J \circ S \circ J=S$ and so $\operatorname{Sym}(M) \cong \mathcal{D}(2 k)$. It is clear that $S$ fixes $P$ and $S\left(\operatorname{orb}\left(Q_{i}\right)\right)=\operatorname{orb}\left(Q_{j}\right), i, j \in\{1,2,3\}$. Since $S$ has order two, we can suppose without loss that $S\left(\operatorname{orb}\left(Q_{1}\right)\right)=\operatorname{orb}\left(Q_{1}\right)$. Thus, $S$ induces an antiholomorphic automorphism $\widetilde{S}$ of the $u$-plane $\bar{M} /\langle J\rangle$ 
that fixes $\infty, 0$ and satisfies $u \circ S=\widetilde{S} \circ u$. It is straightforward to check that, up to a change of variables, $\widetilde{S}(u)=\bar{u}$.

If we define $N=M-\left(\operatorname{orb}\left(Q_{1}\right) \cup \operatorname{orb}\left(Q_{2}\right) \cup \operatorname{orb}\left(Q_{3}\right)\right)$, then

$$
u_{\mid N}: N \rightarrow \mathbb{C}-\{0, c, b\}
$$

is a $2 k$-fold unbranched cyclic covering, and the conformal structure of $N$ determines that of $\bar{M}$. Let $\beta_{i}(t), i=1,2,3$ be counterclockwise circuits around $0, a$ and $b$ respectively, and label $\widetilde{\beta}_{i}(t), i=1,2,3$ as its respective lifts to $N$. Since $J^{m_{i}}\left(Q_{i}\right)=Q_{i}, i=1,2,3$, the end points of $\widetilde{\beta}_{i}(t)$ will differ by a deck transformation of the form $J^{h_{i} m_{i}}$, where $h_{i} \in\left\{1, \ldots, 2 k / m_{i}\right\}$, and $\operatorname{gcd}\left(h_{i}, 2 k / m_{i}\right)=1, i=1,2,3$. Furthermore, the choice of $J$ gives $h_{i} \equiv \pm 1 \bmod \left(2 k / m_{i}\right), \quad i=1,2,3$. Without loss of generality, we put $h_{i} \in\{1,-1\}, i=1,2,3$. The integers $\left\{h_{1}, h_{2}, h_{3}\right\}$ determine the induced map from $\Pi_{1}(\mathbb{C}-\{0, c, b\})$ into $\mathbb{Z}_{2 k}$ whose kernel corresponds to $u_{*}\left(\Pi_{1}(N)\right)$. Consider now the complex curve:

$$
\bar{M}_{1}=\left\{(u, w) \in(\mathbb{C} \cup\{\infty\}): w^{2 k}=u^{m_{1} h_{1}}(u-c)^{m_{2} h_{2}}(u-b)^{m_{3} h_{3}}\right\} .
$$

The cyclic covering defined by the $u$-projection of $\bar{M}_{1}$ has the same properties of $u_{\mid N}$ described above, and so they are equivalent, that is to say, up to conformal transformations:

$$
\bar{M}=\bar{M}_{1}, \quad J(u, w)=(u, \theta w), \text { where } \theta=e^{\frac{\pi i}{k}} .
$$

We denote $[g]_{0},[g]_{\infty}$ as the zero and polar divisor of $g$, respectively. It is evident that $\operatorname{Deg}\left([g]_{0}\right)=\operatorname{Deg}\left([g]_{\infty}\right)=\operatorname{deg}(g)$. For more details see $[\mathbf{F}-\mathbf{K}]$.

If $m_{i}<k, \forall i \in\{1,2,3\}$ then $m_{i} \leq \frac{2 k}{3}$ (recall that $m_{i}$ is a divisor of $2 k)$. Hence, $\sum_{i=1}^{3} m_{i} \leq 2 k$, which contradicts (7). So, at least one of them is equal to $k$. Without loss of generality we suppose $m_{3}=k$. Then, we have $m_{1}+m_{2}=k+1$. If $m_{1}, m_{2} \leq k / 2$ we obtain $m_{1}+m_{2} \leq k$, which is absurd. Therefore, we can assume that $m_{2} \in\left\{k, \frac{2 k}{3}\right\}$ and so we get two possibilities: (i) $m_{1}=\frac{k}{3}+1, m_{2}=\frac{2 k}{3}, m_{3}=k$.

(ii) $m_{1}=1, m_{2}=m_{3}=k$.

Firstly, we study the case (i). In this situation $k$ is a multiple of 3 and $2 k$ is a multiple of $1+k / 3$. Hence, $k \in\{3,6,15\}$. Our hypothesis implies $k=3, m_{1}=m_{2}=2, m_{3}=3$. If $\operatorname{orb}\left(Q_{i}\right) \subset g^{-1}(\infty), \operatorname{orb}\left(Q_{j}\right) \subset g^{-1}(0)$, $\{i, j\}=\{1,2\}$, and as $J^{2}$ is a rotation, and using (8), (9) and (10) then we get $\operatorname{deg}(g)=\operatorname{Deg}\left([g]_{\infty}\right)=4+m_{g}(\infty)+3 l_{1}, \operatorname{deg}(g)=\operatorname{Deg}\left([g]_{0}\right)=4+3 l_{2}$, where $l_{1}, l_{2} \in \mathbb{N}$ and $m_{g}(\infty)$ is the multiplicity of $g$ at $\infty$. It is easy to deduce that $l_{1}, l_{2}>0$ and so $\operatorname{deg}(g)>5$. If $\operatorname{orb}\left(Q_{1}\right) \cup \operatorname{orb}\left(Q_{2}\right)$ is contained in either 
$g^{-1}(0)$ or $g^{-1}(\infty)$, then similar arguments lead to $\operatorname{deg}(g) \geq 8+6 l>5, l \in \mathbb{N}$. A discussion about the excluded cases $k=6,15$ can be found in Remark 1 below.

Now consider case (ii).

First suppose $k$ is odd.

Assume that $J$ is a rotation. If $\operatorname{orb}\left(Q_{1}\right) \subset g^{-1}(\infty)$ then using (8) and (9) we get:

$$
\operatorname{deg}(g)=\operatorname{Deg}\left([g]_{\infty}\right)=2 k-1+m_{g}(\infty)+2 l k>2 k-1, l \in \mathbb{N} .
$$

If $\operatorname{orb}\left(Q_{1}\right) \subset g^{-1}(0)$, then using similar arguments, we have

$$
\operatorname{deg}(g)=\operatorname{Deg}\left([g]_{0}\right) \geq 2 k-1+2 k l, l \in \mathbb{N} .
$$

If $\operatorname{deg}(g)=\operatorname{Deg}\left([g]_{0}\right)=2 k-1$ then $l=0$ and $\operatorname{so} \operatorname{orb}\left(Q_{2}\right) \cup \operatorname{orb}\left(Q_{3}\right) \subset g^{-1}(\infty)$. Hence from (8) and (9) $\operatorname{deg}(g)=\operatorname{Deg}\left([g]_{\infty}\right) \geq 2 k+m_{g}(\infty)$, which is absurd.

Suppose now $J$ is a rotation followed by a symmetry and suppose $\operatorname{orb}\left(Q_{1}\right) \subset$ $g^{-1}(0)$, then using once again (8) and (10) we obtain $\operatorname{deg}(g)=\operatorname{Deg}\left([g]_{0}\right)=$ $k-1+2 l k, l \in \mathbb{N}$. Jorge-Meeks formula (3) implies $l>0$. If $\operatorname{orb}\left(Q_{1}\right) \subset$ $g^{-1}(\infty)$, from (8) and (10) $\operatorname{Deg}\left([g]_{0}\right)$ is a multiple of $2 k$. In both cases $\operatorname{deg}(g)>2 k-1$.

Assume now that $k$ is even. If $J$ is a rotation, the same proof of the case when $k$ is odd gives $\operatorname{deg}(g)>2 k-1$. Now, suppose $J$ is a rotation followed by a symmetry. If $\operatorname{orb}\left(Q_{2}\right) \cup \operatorname{orb}\left(Q_{3}\right)$ is contained in either $g^{-1}(0)$ or $g^{-1}(\infty)$, then (8) and (9) imply $\operatorname{deg}(g) \geq 2 k$. When $\left\{g\left(Q_{2}\right), g\left(Q_{3}\right)\right\}=\{0, \infty\}$, taking into account that the multiplicity of $Q_{1}$ as either zero or pole of $g$ is $k-1+2 l k$, $l \in \mathbb{N}$ (see (10)) we get:

$$
\operatorname{deg}(g) \geq 2 k-1
$$

and the equality holds if and only if $l=0$ and

$$
[g]_{0}=Q_{1}^{k-1} Q_{i} \cdots J^{k-1}\left(Q_{i}\right),[g]_{\infty}=P^{k-1} Q_{j} \cdots J^{k-1}\left(Q_{j}\right)
$$

where $\{i, j\}=\{2,3\}$. Up to changes of variables and relabelings, we can suppose $i=3$ and $j=2$. Labeling $u=t^{k}$, i.e., $t=w^{2}(u-c) /(u-b)$, up to a biholomorphism, we obtain:

$$
\begin{gathered}
\bar{M}=\left\{(t, w) \in \overline{\mathbb{C}}^{2}: w^{2}=\frac{t\left(t^{k}-b\right)}{t^{k}-c}\right\} \\
g=A t^{k / 2-1} w, \eta g=B t^{k / 2-1} d t .
\end{gathered}
$$

Up to scaling and rigid motions, $A \in \mathbb{R}, B \in \mathbb{C},|B|=1$. 
There exist two possibilities: $S\left(\operatorname{orb}\left(Q_{2}\right)\right)=\operatorname{orb}\left(Q_{3}\right)$ and $S\left(\operatorname{orb}\left(Q_{i}\right)\right)=$ $\operatorname{orb}\left(Q_{i}\right), i \in\{2,3\}$. In the first case, $\widetilde{S}(c)=\bar{c}=b$ and without loss of generality we can suppose

$$
S(t, w)=(\bar{t}, \bar{t} / \bar{w})
$$

Looking at the Weierstrass data above, $S$ does not correspond to any symmetry of the surface. We deduce then that $S\left(\operatorname{orb}\left(Q_{i}\right)\right)=\operatorname{orb}\left(Q_{i}\right), i \in$ $\{1,2,3\}$ and so $c, b \in \mathbb{R}-\{0\}$ and $S(t, w)=(\bar{t}, \bar{w})$. Up to a change of variables, we can assume that $c=1$.

If $b>0$, making $a=\sqrt[k]{b}>0$, Theorem 1 leads to the surface $M_{k}$.

If $b<0$, write $a=\sqrt[k]{-b}>0$. We label $\gamma_{1}$ as an oriented simple closed curve in the $t$-plane whose winding numbers around 0 and 1 are -1 and around $a e^{\frac{(2 j+1) \pi i}{k}}, e^{\frac{2 l \pi i}{k}}$, are $0, j=0, \ldots, k-1, l=1, \ldots, k-1$. Furthermore we assume that $\gamma_{1}$ satisfies $\gamma_{1}(0) \in \mathbb{R}, \gamma_{1}(0)>1$. In the same way we label $\gamma_{2}$ as an oriented simple closed curve in the $t$-plane whose winding numbers around $0, a e^{\frac{\pi i}{k}}$ are -1 and the winding numbers around $a e^{\frac{(2 j+1) \pi i}{k}}, e^{\frac{2 l \pi i}{k}}$, are $0, j=1, \ldots, k-1, l=0, \ldots, k-1$. Suppose $\operatorname{Arg}\left(\gamma_{2}(0)\right)=\frac{\pi}{k}$ and $\left|\gamma_{2}(0)\right|>a$. Let $c_{i}$ be the lift of $\gamma_{i}$ to $\bar{M}, i=1,2$, with initial conditions $\operatorname{Arg}\left(w\left(c_{1}(0)\right)\right)=0, \operatorname{Arg}\left(w\left(c_{2}(0)\right)\right)=\frac{\pi}{2 k}$. Using the same notation as in Theorem 1, we write $\tau_{1}=\frac{d t}{w}$ and $\tau_{2}=t^{k-2} w d t$. Hence, it is not hard to check that

$$
\int_{c_{1}} \tau_{1}=2 i F_{1}, \int_{c_{1}} \tau_{2}=-2 i G_{1}, \int_{c_{2}} \tau_{1}=-2 i e^{\frac{\pi i}{2 k}} F_{2}, \int_{c_{2}} \tau_{2}=-2 i e^{-\frac{\pi i}{2 k}} G_{2}
$$

where $F_{j}, G_{j}>0, j=1,2$. If we suppose $\phi_{1}, \phi_{2}$ has no real periods then

$$
\frac{B}{A} \int_{c_{j}} \tau_{1}=A \bar{B} \overline{\int_{c_{j}} \tau_{2}}, j=1,2 .
$$

If $j=1$ then $B^{2}=1$ and similarly $j=2$ implies $B^{2}=-1$, which is absurd.

Second case: $\operatorname{ord}(J)=4 k$.

Taking into account (7) then $s=2$ and $m_{1}+m_{2}=2 k+1$. It is obvious that $m_{1}, m_{2} \leq k$ leads to a contradiction. Since $4 k$ is a multiple of $m_{i}$, $i=1,2$, without loss of generality $m_{1} \in\left\{2 k, \frac{4 k}{3}\right\}$. Hence $m_{1}$ is even and so $J^{m_{1}}$ is a rotation. If $Q$ is a fixed point of $J^{m_{1}}$ then the multiplicity of $Q$ as zero or pole of $g$ is $\frac{\operatorname{ord}(J)}{m_{1}} l_{1}-1$, where $l_{1} \in \mathbb{N}$ (see (9)). Taking (8) into account one has

$$
\operatorname{deg}(g) \geq \operatorname{ord}(J) l_{1}-m_{1} \geq \operatorname{ord}(J)-m_{1} \geq 2 k>2 k-1 .
$$

This completes the proof. 
Remark 1. If $k \in\{6,15\}$ then it is possible to find algebraic curves $\bar{M}$ of genus $k$, different to $\bar{M}_{k}$, and Weierstrass data $(g, \eta)$ on $\bar{M}-\{P\}$ with suitable symmetry such that $\operatorname{deg}(g) \leq 2 k-1$. The analysis of the period problem associated to the following Weierstrass data is a little different to the one in Section 3, and it is still open.

For $k=6$, consider $\left(M=\bar{M}-\{P\}, g_{i}, \eta_{i}\right), \mathrm{i}=1,2$, where

$$
\begin{aligned}
& \bar{M}=\left\{(u, w) \in \mathbb{C} \cup\{\infty\}: w^{12}=\frac{u^{3}(u-1)^{4}}{(u-b)^{6}}\right\}, b \in \mathbb{R}-\{0,1\}, P=(\infty, \infty) \\
& g_{1}=A \frac{w^{5}(u-b)^{2}}{u(u-1)}, \quad \eta_{1} g_{1}=B \frac{u(u-1)^{2}}{w^{6}(u-b)^{3}} d u \\
& g_{2}=A \frac{w(u-b)}{(u-1)}, \quad \eta_{2} g_{2}=B \frac{u(u-1)^{2}}{w^{6}(u-b)^{3}} d u .
\end{aligned}
$$

For $k=15$, take $(M=\bar{M}-\{P\}, g, \eta)$, where

$$
\begin{gathered}
\bar{M}=\left\{(u, w) \in \mathbb{C} \cup\{\infty\}: w^{30}=\frac{u^{6}(u-b)^{15}}{(u-1)^{10}}\right\}, b \in \mathbb{R}-\{0,1\}, P=(\infty, \infty) \\
g=A \frac{w^{14}(u-1)^{4}}{u^{2}(u-b)^{7}}, \quad \eta g=B \frac{u^{3}(u-b)^{7}}{w^{15}(u-1)^{5}} d u .
\end{gathered}
$$

Corollary 1. The only complete orientable genus two minimal surface in $\mathbb{R}^{3}$ with total curvature $-12 \pi$ and eight symmetries is the Chen-Gackstatter example.

Proof. From Huber Theorem $[\mathbf{H}], M$ is conformally equivalent to $\bar{M}-\left\{P_{1}, \ldots\right.$, $\left.P_{r}\right\}$, where $\bar{M}$ is a compact genus 2 Riemann surface. Furthermore from Jorge-Meeks formula (3) $r \in\{1,2\}$. If $r=1$ then $\nu_{1}=3$ and $r=2$ gives $\nu_{1}=\nu_{2}=1$. The second possibility leads to the catenoid (see [Sch]) which is absurd. From Theorem 2 the first one corresponds to the Chen-Gacksttater genus two example.

\section{References}

[B] D. Bloss, Elliptische funktionen und vollständige Minimalflächen, $\mathrm{Ph}$. Thesis, Freien Universität Berlin, 1989.

[C-G] C.C. Chen and F. Gackstatter, Elliptische und hyperellipsche Funktionen und vollständige Minimalflächen vom Enneperschen Typ, Math. Ann., 259 (1982), 359-369. 
[C] C. Costa, Example of a complete minimal immersion in $\mathbb{R}^{3}$ of genus one and three embedded ends, Bol. Soc. Bras. Math., 15 (1984), 47-54.

[F-K] H.M. Farkas and I. Kra, Riemann surfaces, Grad. Texts in Math., 72, Springer Verlag, Berlin, 1980.

[H-M] D. Hoffman and W.H. Meeks III, Embedded minimal surfaces of finite topology, Annals of Mathematics, 131 (1990), 1-34.

[H] A. Huber, On subharmonic functions and differential geometry in the large, Comment. Math. Helv., 32 (1957), 13-72.

[J-M] L. Jorge and W.H. Meeks, The topology of complete minimal surfaces of finite total gaussian curvature, Topology, 22 (1983), 203-221.

[K] H. Karcher, Construction of minimal surfaces, Surveys in Geometry, 1-96, University of Tokyo, 1989, (also: Lecture Notes No. 12, SFB256, Bonn, 1989).

[L1] F.J. Lopez, The classification of complete minimal surfaces with total curvature greater than $-12 \pi$, Trans. Amer. Math. Soc., 334(1) (1992), 49-74.

[L2] Complete minimal surfaces with one end, preprint.

[M-R] F. Martin and D. Rodriguez, A new approach to the construction of complete minimal surfaces derived from the genus two Chen-Gackstatter example, Illinois J. Math., 41(2) (1997), 171-192.

[O] R. Osserman, A survey of minimal surfaces, Dover Publications, New York, second edition, 1986.

[S] K. Sato, Constructions of higher genus minimal surfaces with one end and finite total curvature, Tôhoku Math. J., 48 (1996), 229-246.

[Sch] R. Schoen, Uniqueness, symmetry and embeddedness of minimal surfaces, Journal of Differential Geometry, 18 (1983), 791-809.

[Str] K. R. Stromberg, An introduction to classical real Analysis, Wadsworth International Group, Belmont, California, 1981.

[T] E.C. Thayer, Higher genus Chen-Gackstatter surfaces and the Weierstrass representation for infinite genus surfaces, to appear in Jour. of Experimental Mathematics, 4(1) (1995), 11-31.

[WW] M. Weber and M. Wolf, Minimal surfaces of least total curvature, preprint.

Received November 11, 1996 and revised April 10, 1997. This research was partially supported by DGICYT Grant No. PB94-0796.

\author{
Departamento de Geometria y Topologia \\ UNIVERSIDAD DE GRANADA \\ 18071 - GRANADA (SPAIN) \\ E-mail address: fjlopez@goliat.ugr.es \\ fmartin@goliat.ugr.es \\ drodrig@goliat.ugr.es
}

